\title{
ELISA Measurement of Interferons
}

\author{
T.L. Garrison, F. Hung, L. Izotova, B. Schwartz, T. Lavoie, and F.V. Lee-Own \\ PBL Biomedical Laboratories, Piscataway, NJ, USA
}

BioTechniques 33:S98-S100 (October 2002)

\section{INTRODUCTION}

Since the first descriptions of the utility of immunoassays using enzymes rather than the then-dominant radioisotopic labeling required for RIAs $(1-4,13)$ and the subsequent use of 96 -well microplates $(14,15)$, ELISAs formatted in 96-well microplates have become widely used for both research assays and routine clinical testing. Some of the rationales for using enzyme conjugates as opposed to radiolabels include the facts that the assays can be performed in any laboratory (while counting of radioactivity requires radioisotope counting equipment), the measurement of enzyme activity is less time consuming than counting radioactivity, and enzyme conjugates have greater useful shelf-life than their radiolabeled counterparts (6-12 months vs. 6-8 weeks, respectively). ELISAs are non-isotopic and versatile, and the current assay products are robust, sensitive, specific, and, in some cases, the only way to measure the analyte in question.

ELISAs are widely used in both the research and clinical laboratory, either because no other type of assay system is as feasible and/or economic or because they are often the most effective and suitable of the possible analytical methods. These assays can be done practically anywhere. They are precise and reliable, can be run fully automated, semi-automated, or in single-plate manual format, and are capable of having detection limits in the pico- or nanogram range if designed with high-quality reagents. The analytes that can be detected by ELISAs range from small haptens and drugs, such as digoxin, to large proteins, such as ferritin.

The analytical sensitivity of an ELISA depends largely on the activity of the enzyme-labeled reagent. If an enzyme with a high turnover rate is used, such as HRP, then very small amounts of enzyme can be detected. Other enzymes that have been used in ELISAs include alkaline phosphatase, $\beta$-galactosidase, glucose6-phosphate dehydrogenase, urease, and luciferase (6). In addition, the stability and quality of the component reagents and their compatibility in the assay format normally determine the robustness of the ELISA. The more highly purified the immunoglobulin that is used, whether polyclonal or monoclonal, the better the resultant assays are performed and reproduced. If high-quality hyperimmune polyclonal antiserum is available, then it is possible that it can be diluted and used for capture antibody immobilized directly onto microplate wells or, in sandwich assays, as detection antibody. In this example, a subsequent enzyme-conjugated anti-species antibody can used to generate a signal. More commonly though, high specific activity immunoglobulin is purified by conventional, Protein A, or antigen affinity chromatography and used for producing solid-phase capture antibody or detection antibody for direct conjugation with enzyme. High-quality antibody components are very robust and can be configured in some assay formats to be stable for up to 24 months at ambient temperatures.

ELISAs have been used for applications ranging from highthroughput, research-oriented screening assays to single use in vitro diagnostic over-the-counter test devices. Additionally, the principles of the technology have been applied to the construction of all manner of binding assays: antigen, ligand, antibody, receptor, or binding molecule can be immobilized as a solid phase, which in turn can capture of the appropriate ligand- or target-binding molecule that is conjugated to an enzyme, and a subsequent colorimetric, fluorimetric, or luminescent signal generation monitors the reaction. Ligand-binding assays for high-throughput screening drug and target discovery are assays constructed with ligands and receptor-binding molecules, which can be considered the functional equivalents of antigens and antibodies, respectively.

\section{Use of ELISA for Interferon Measurement}

Interferons are a family of antiviral substances that confer upon cells the ability to resist viral infection. Interferon was first described in 1957 (7), and it is now known that there are several distinct interferon species that can be put into two main subtypes, Type I interferon (IFN- $\alpha$, IFN- $\beta$, IFN- - , IFN- $\omega$, and IFN- $\tau$ ) and Type II (IFN- $\gamma$ ). Additionally, the human $\alpha$-interferons consist of multiple individual species (8).

Interferons have been detected and measured historically by their unique ability to confer viral resistance to cells. Assays for 
interferons that measure their antiviral properties are known as cytopathic effect inhibition assays or antiviral assays $(5,11)$.

Any molecule with a functional biological activity can be measured by its activity, by its mass (or mass equivalents), or a by combination of both (specific activity, usually expressed as units of activity/mg mass). In the case of interferon, antiviral activity measurements traditionally defined the unique antiviral properties of the molecule $(5,11)$, and mass-based measurements were first developed using RIAs (12). Measurements of interferon antiviral activity give no information about the purity or mass of material present. Similarly, mass or mass-equivalent measurements do not give information about the antiviral activity, state of denaturation, or purity. Once it was shown that monoclonal antibodies could be developed that reacted preferentially with native (undenatured) interferons, it was then logical to use them to construct immunoassays that detected native interferons, and they could thus be used to replace the antiviral assay for biologically active interferon (10).

The use of an ELISA will usually give results for interferons in mass equivalents. The validity of the mass equivalent obtained will be based on the specificity of the antibodies used in the kits. However, if the antibody reagents used are selected appropriately, then the resultant ELISA can be constructed to detect only biologically active interferon; thus, the ELISA measurements will also reflect antiviral activity.

PBL Biomedical Laboratory's (Piscataway, NJ, USA) range of ELISA kits detect human IFN- $\alpha$, IFN- $\beta$, and IFN- $\omega$, mouse IFN- $\alpha$, and mouse and rat and IFN- $\gamma$. Additionally, PBL has ELISA kits for IFN- $\alpha$ that detect the majority of the IFN- $\alpha$ subtypes (Human Interferon Alpha Multispecies Kit) and also a kit suitable for use with human serum samples (Human Interferon Alpha Serum Samples Kit). The test kits are formatted on microplates, which are used for the solid phase. All are in the "strip-well" or "breakaway" format, allowing the user the flexibility of using as many wells as needed, and feature sequential addition sandwich ELISAs, using either monoclonal-monoclonal or monoclonal-polyclonal antibodies to sandwich the interferon. Typically, the solid phase is purified monoclonal antibody immobilized on the microplate well, and the detection antibody

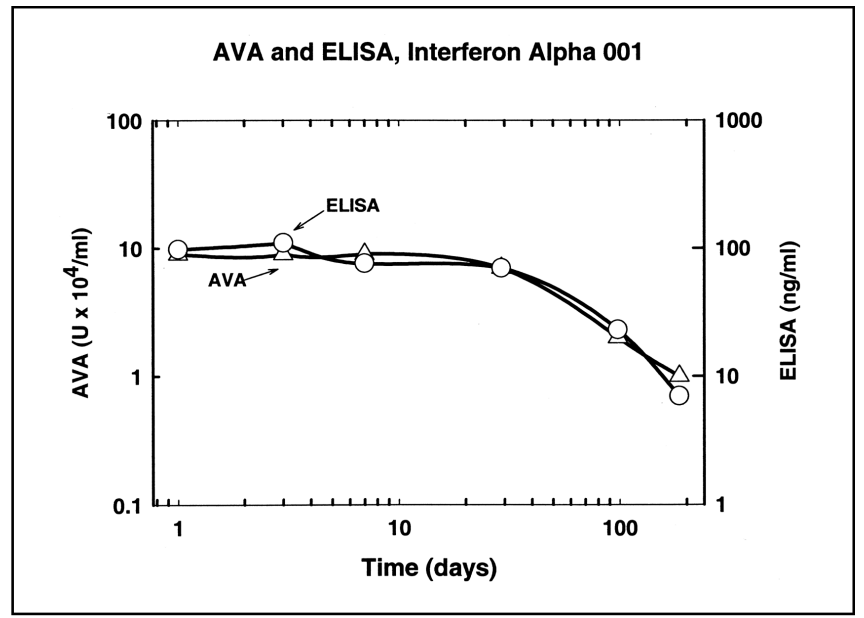

Figure 1. Antiviral Activity (AVA) and ELISA measurements for a preparation of interferon $\alpha 001$ after incubation at $37^{\circ} \mathrm{C}$ for various times. is either hyperimmune polyclonal antiserum (in which case an anti-species antibody conjugated to HRP is used) or a monoclonal antibody (in which case the detection antibody is biotinylated and used with a streptavidin-labeled HRP conjugate). Tetra-methyl benzidine is the substrate in all kits. All reagents are supplied in liquid form, together with appropriate diluents and instructions for use. These assay designs and configuration of reagents have allowed PBL to develop a series of robust assays with sensitivities in the picogram range and with the unique ability to detect and differentiate biologically active interferons.

To demonstrate that PBL's Human Interferon Alpha Multispecies ELISA Kit measures biologically active interferon, the kit was used to monitor a preparation of interferon $\alpha$ that was stored at $37^{\circ} \mathrm{C}$ for a period of 185 days.

\section{MATERIALS AND METHODS}

Human interferon $\alpha 001$ (Hu-IFN- $\alpha 001$ ), a recombinant polypeptide having interferon activity and a sequence derived from diseased leukocytes, was prepared and purified as described using ammonium sulfate precipitation and FPLC chromatography (9). It was assayed for antiviral activity on human and bovine cells with vesicular stomatitis virus (VSV) using the international standard Gxa01-901-535 as a reference $(5,11)$. The Hu-IFN- $\alpha 001$ was diluted to about $1 \times 10^{5}$ units in a buffer matrix of PBS containing $20 \mathrm{mM}$ Tris- $\mathrm{HCl}, \mathrm{pH} 8.0,0.1 \%$ BSA, $100 \mathrm{mM}$ calcium chloride, $5 \%$ glycerol, $200 \mathrm{mM}$ sodium chloride, and incubated at $37^{\circ} \mathrm{C}$ for 185 days. Portions of $30 \mu \mathrm{L}$ were removed under sterile conditions after 1,3, 7, 29, 98, and 185 days and stored at $-20^{\circ} \mathrm{C}$ until assayed. Parallel measurements of antiviral activity (biological activity) and ELISA were performed. The antiviral assay, a conventional cytopathic effect inhibition assay with Madin-Darby bovine kidney cells challenged with VSV, was read visually after staining of the plates with $100 \mu \mathrm{L} /$ well of $0.5 \%$ crystal violet in methanol, the titer being $50 \%$ cytopathic effect-where half the cells are dead and half are alive $(5,11)$. ELISA measurements were carried out according to the package insert for the Human Interferon Alpha Multispecies ELISA Kit. All antiviral and ELISA measurements were performed in duplicate at the end of the 185-day period.

\section{RESULTS AND DISCUSSION}

Figure 1 shows that for $\mathrm{Hu}$-IFN- $\alpha 001$ under the conditions studied the antiviral activity and ELISA activity paralleled each other over the 185-day time period. Both assays showed decreasing values of antiviral activity and ELISA immunoreactivity. The manner of the correlation indicates that the kit recognizes active and $\mathrm{Hu}-\mathrm{IFN}-\alpha 001$ and not the inactive material produced from the interferon over time, the interferon activity presumably being lost because of this denaturation.

Thus, we have shown that careful selection of the appropriate antibodies is responsible for the kit performance and, in particular, the ability of the kit to detect and measure active interferon. The correlation between the antiviral activity of the preparations and the ELISA immunoreactivity can be due to several different factors involving the specificity of antibody 


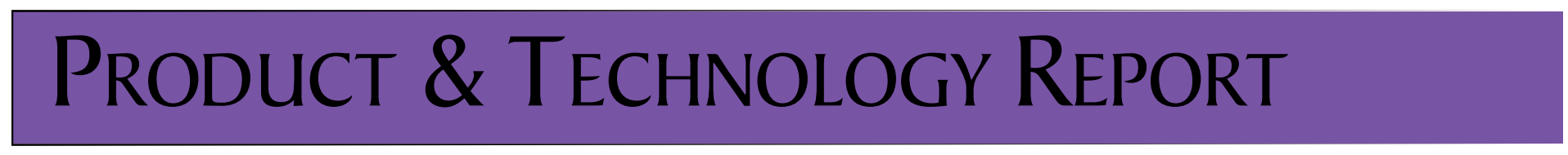

binding to its epitope on the interferon. The kit antibodies, or at least one of them, may bind to an epitope on the interferon that is necessary for bioactivity when the interferon is in an active undenatured state. That epitope could either be linear, based on the primary structure, or conformational. However, either way it is present in undenatured and bioactive interferon. When the interferon is denatured, this epitope will be absent because of perhaps masking and/or reconfiguration of the peptide chains. Alternatively, another possibility is that the epitope representing the antibody-binding site may be present on the undenatured interferon but not involved in any respect with the bioactivity of the molecule. However, when the interferon is denatured, this epitope is either obscured or eliminated by the reconfiguration of the peptide chain, which corresponds to a loss of bioactivity, and antibody binding cannot take place.

In summary, the Human Interferon Alpha Multispecies ELISA kit measures the activity of $\mathrm{Hu}-\mathrm{IFN}-\alpha 001$. Although not shown here, the kit is also useful for detecting almost all human interferon $\alpha$ species.

\section{ACKNOWLEDGMENTS}

We would like to thank Sidney Pestka for his insightful discussions and for helping to revise this manuscript. We are especially grateful to Jim Siverly and William A. Clark for their help in analyzing the data. We also thank Kristen A. Foster for her preparation of reagents and computer expertise.

\section{REFERENCES}

1.Engvall, E., K. Jonsson, and P. Perlman. 1971. Enzyme-linked immunosorbent assay (ELISA) quantitative assay of immunoglobulin G. Immunochemistry 8:871-874.

2.Engvall, E., K. Jonsson, and P. Perlman. 1971. Enzyme-linked immunosorbent assay. II. Quantitative assay of protein antigen, immunoglobulin-G, by means of enzyme-labelled antigen and antibody-coated tubes. Biochim. Biophys. Acta 251:427-434.

3.Engvall, E. and P. Perlman. 1971. Enzyme-linked immunosorbent assay, ELISA. p. 553-556. In H. Peeters (Ed.), Protides of the Biological Fluids. Proceedings of the Nineteenth Colloquium. Pergamon Press, Oxford.

4.Engvall, E. and P. Perlman. 1972. Enzyme-linked immunosorbent assay, ELISA. III. Quantitation of specific antibodies by enzyme-labeled anti-immunoglobulins in antigen-coated tubes. J. Immunol. 109:129-135.

5.Familletti, P.C., S. Rubinstein, and S. Pestka. 1981. A convenient and rapid cytopathic effect inhibition assay for interferon. p. 387-394. In S. Pestka (Ed.) Methods in Enzymology, vol. 78, Academic Press, New York.

6.Gosling, J. 1990. A decade of development in immunoassay methodology. Clin. Chem. 36:1408-1427.

7.Isaacs, A. and J. Lindenmann. 1957. Virus interference. I. The interferon. Proc. Royal Society London Ser. B. 147:258-267.

8.Pestka, S. 1997. The human interferon alpha species and hybrid proteins. Semin. Oncol. 24:S9-4-S9-17.

9.Pestka, S., inventor; PBL Biomedical Laboratories, assignee. 1998. Human leukocyte interferon HU-IFN- $\alpha 001$.US Patent 5789551.

10.B. Kelder, J.A. Langer, and T. Staehelin. 1983. Monoclonal antibodies can discriminate between some active and inactive forms of leukocyte interferon. Arch. Biochem. Biophys. 224:111-116.

11.Rubinstein, S., P.C. Familletti, and S. Pestka. 1981. Convenient assay for interferons. J. Virol. 37:755-758.

12.Staehelin, T., C. Stähli, D.S. Hobbs, and S. Pestka. 1981. A rapid quantitative assay of high sensitivity for human leukocyte interferon with monoclonal antibodies, p.589-585. In S. Pestka (Ed.) Methods in Enzymology, vol. 79, Academic Press, New York.
13.Van Weeman, B.K. and A.H.W.M. Schuurs. 1971. Immunoassay using antigen-enzyme conjugates. FEBS Lett. 15:232-235.

14. Voller, A. and D.E. Bidwell. 1975. A simple method for detecting antibodies to rubella. Br. J. Exp. Pathol. 56:338-339.

15.Voller, A. and D.E. Bidwell. 1976. Enzyme-immunoassays for antibodies in measles, cytomegalovirus infections and after rubella vaccination. Br. J. Exp. Pathol. 57:243-247.

Address correspondence to:

Dr. F. Victor Lee-Own

PBL Biomedical Laboratories

131 Ethel Road West, Suite 6

Piscataway, NJ, USA

e-mail:vlo@pblbio.com 